- In the oral cavity paraformaldehyde agents are used both as disinfectants and to devitalise inflamed pulps when local anaesthesia is ineffective.

- After root canal treatment with paraformaldehyde containing paste, formaldehyde is gradually released into the oral cavity.

- Formaldehyde released through dentine has a destructive effect on periodontal and bone tissues.

- Paraformaldehyde use in dental practice should be strongly discouraged.

\title{
Maxillary bone necrosis following the use of formaldehyde containing paste: management and case series
}

\author{
S. Tortorici, ${ }^{1}$ F. Burruano ${ }^{2}$ and P. Difalco ${ }^{3}$
}

Paraformaldehyde is used both as a disinfectant and to devitalise inflamed pulps when local anaesthesia is ineffective. Despite the clinical benefits, paraformaldehyde is not confined to the pulp, but penetrates the dentine and is gradually released as formaldehyde. This case series describes the effects on periodontal and bone tissues of paraformaldehyde used as a devitalising or disinfectant agent.

\section{INTRODUCTION}

Many of the medicaments used historically in root canal treatment have been shown to be cytotoxic. Among these substances an important role was played by paraformaldehyde pastes. Paraformaldehyde is a precursor of formaldehyde and releases formaldehyde gas which may be used as a disinfectant. Today formaldehyde is used in a wide variety of products. These products include skin and hair care products, cosmetics, certain cleaning products, medications, permanent press textiles and disinfectants. ${ }^{1}$

In the oral cavity paraformaldehyde agents such as Toxavit ${ }^{\varpi}$ are used both as disinfectants and to devitalise inflamed pulps when local anaesthesia is

\footnotetext{
Professor of Maxillofacial Surgery, Department of Stomatological Science, G. Messina University of Palermo, Palermo, Italy; ${ }^{2}$ Professor of Maxillofacial Surgery, Department of Stomatological Science, G. Messina University of Palermo, Palermo, Italy; ${ }^{{ }^{*}}$ Department of Stomatological Science, G. Messina University of Palermo, Palermo, Italy

${ }^{*}$ Correspondence to: Dr Paolo Difalco

Email:paolodifalco@tin.it
}

\section{Refereed Paper}

Accepted 24 April 2007

DOI: $10.1038 /$ bdj.2007.995

${ }^{\circledR}$ British Dental Journal 2007; 203: 511-512 ineffective. ${ }^{2}$ Despite the clinical benefits paraformaldehyde is not confined to the pulp, but penetrates through dentine and is gradually released as formaldehyde. ${ }^{1,2}$ Formaldehyde released through dentine has a destructive effect on periodontal and bone tissues. This article describes the effects on the periodontal and bone tissues of the paraformaldehyde used as a devitalising or disinfectant agent.

\section{CASE 1}

A 55-year-old man with a history of severe pain in the right mandibular molar region was referred for assessment. The patient reported symptoms of irreversible pulpitis from tooth 46 around one month previously. His general dental practitioner was unable to obtain adequate anaesthesia to extirpate the pulp from this tooth and applied paraformaldehyde paste to effect devitalisation. Subsequently the patient stated that he experienced severe pain, the tooth became mobile and there was a swelling in the lower right molar area.

After a week, teeth 46 and 47, which had become mobile, were extracted and antibiotics and analgesics were prescribed. The patient had symptomatic improvement, but after seven days necrosis of both buccal and lingual gingivae was evident. These tissues were excised by the same dentist. Subsequently the patient noticed denudation of the bone. A month after his initial complaint, the patient visited our clinic. On oral examination, there was severe loss of the alveolar mucosa around the extraction sockets and alveolar bone was exposed.

A diagnosis of chemical osteonecrosis of the mandible was made (Fig. 1). Disinfection of the mouth was performed with chlorhexidine $0.2 \%$ and, under local anaesthesia, the necrotic bone was removed locally and curettage of the cavity was carried out. The surgical site was irrigated with sterile physiological saline. The flap was closed with a 3/0 silk suture and haemostasis was obtained. The patient was advised to use a chlorhexidine $2 \%$ gel, twice a day in the sutured area. Healing progressed uneventfully and sutures were removed a week later.

\section{CASE 2}

A 30-year-old male patient was referred to our department for a bony sequestrum associated with the interproxi- 
mal area between teeth 46 and 47 . Two months previously, he had visited his dentist due to the severe pain associated with tooth 46. The practitioner elected to perform root treatment of this tooth, which had showed signs of pulpitis, with a paraformaldehyde paste, because the patient reported hypersensitivity to the local anaesthetic articaine. Shortly after the placement of the paraformaldehyde paste, the patient experienced severe pain and after a week he noticed denudation of bone around the tooth.

Clinical examination revealed extensive loss of the buccal attached gingiva and alveolar mucosa around the interproximal socket between teeth 46 and 47 . In this area the alveolar bone was exposed (Fig. 2). Teeth 46 and 47 were mobile. A periapical radiograph revealed the presence of a sequestrum between teeth 46 and 47 (Fig. 3). Disinfection of the mouth was performed with chlorhexidine $0.2 \%$ and, under local anaesthesia with mepivacaine, teeth 46 and 47 were extracted. Necrotic bone was removed locally and curettage of the cavity was carried out. The surgical site was irrigated with sterile physiological saline. The flap was closed with a 3/0 silk suture and haemostasis was obtained. The patient was advised to use a chlorhexidine $2 \%$ gel, twice a day in the sutured area. Healing progressed uneventfully and sutures were removed a week later.

\section{CASE 3}

A 50-year-old female was referred to our department by her local practitioner. Her presenting complaint was bony sequestrum associated with previous tooth extraction in the right maxillary molar region. This sequestrum had been present for around three weeks. The patient revealed that a month previously, she had been treated for acute periodontitis of the upper right molar. Treatment was carried out with a paraformaldehyde-containing root canal disinfectant and zinc oxide eugenol cement. Clinical examination revealed that the patient was now edentulous. A sequestrum was noted in the upper right maxillary oral region (Fig. 4). Disinfection of the mouth was performed with chlorhexidine $0.2 \%$ and the sequestrum was removed under local anaesthesia. Then the surgical site was irrigated with sterile physiological saline. The flap was easily closed with a $3 / 0$ resorbable suture (Fig. 5). The suture

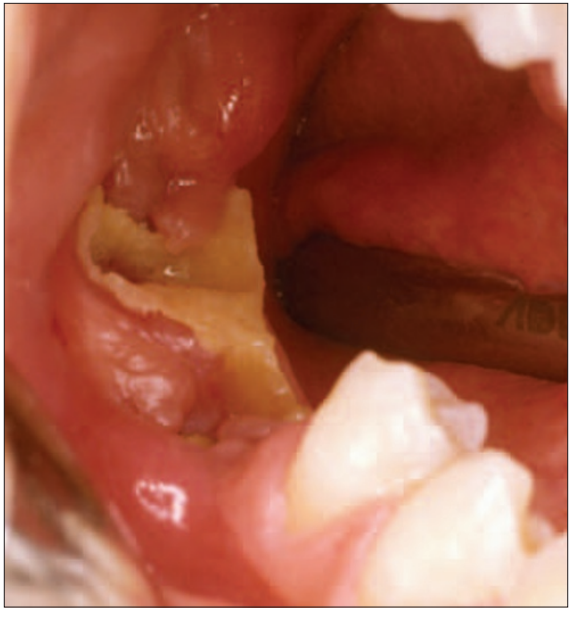

Fig. 1 Severe loss of the alveolar mucosa around the extraction sockets and exposure of the alveolar bone

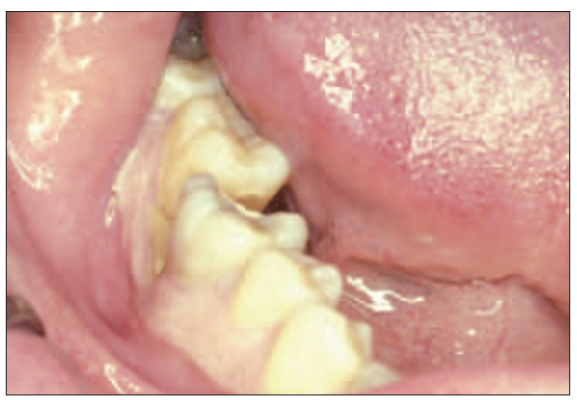

Fig. 2 Extensive loss of the buccal attached gingiva and alveolar mucosa around the interproximal socket between teeth 46 and 47

was removed a week later and healing progressed uneventfully .

\section{DISCUSSION}

The primary cause of pulpal pathosis is bacterial infection. Without the presence of bacteria in the root canal system, periradicular inflammation will not develop or persist. ${ }^{3}$ Consequently, the purpose of root canal treatment is the elimination of bacteria and their substrates from the pulp canal system. During this treatment the pulp is removed and the root canal is cleaned and shaped. Paraformaldehyde is a chemical substance, which has been used during dental treatment, especially for devitalisation and root canal disinfection when local anaesthesia is ineffective. In the oral cavity paraformaldehyde containing paste can provoke both gingival and maxillary bone necrosis. After root canal treatment with paraformaldehyde containing paste, formaldehyde is gradually released into the oral cavity. ${ }^{1}$

Formaldehyde is a well-known allergen and it can cause contact dermatitis mediated by delayed-type hypersensitivity. Formaldehyde has also, on rare occa-

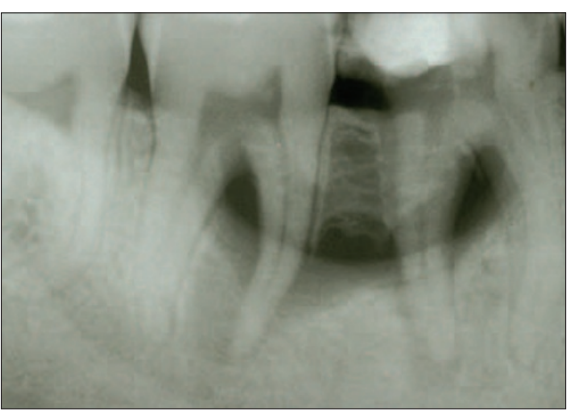

Fig. 3 A periapical radiograph revealed the presence of a sequestrum between teeth 46 and 47

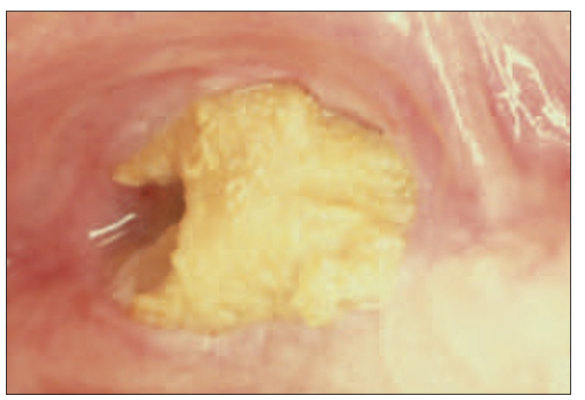

Fig. 4 A sequestrum was noted in the upper right maxillary oral region

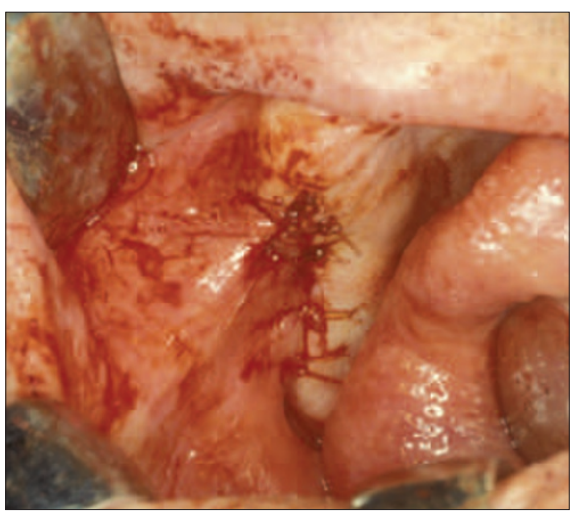

Fig. 5 The flap was easily closed with a 3/0 resorbable suture

sions, mediated Type-I hypersensitivity (anaphylaxis). ${ }^{1}$ Previously, where local anaesthetic was ineffective, paraformaldehyde was used as a devitalising agent. Today, however, effective local anaesthetics and the availability of other sedative agents, eg Ledermix paste, have rendered the use of paraformaldehyde obsolete. Its use in dental practice is therefore to be strongly discouraged in all instances.

1. Kunisada M, Adachi A, Asano H, Horikawa T. Anaphylaxis due to formaldehyde released from root-canal disinfectant. Contact Dermatitis 2002; 47: 215-218.

2. Ozgoz M, Yagiz H, Cicek Y, Tezel A. Gingival necrosis following the use of a paraformaldehydecontaining paste: a case report. Int Endod J 2004; 37: 157-161.

3. Lin L M, Rosenberg P A, Lin J. Do procedural errors cause endodontic treatment failure? J Am Dent Assoc 2005; 136: 187-193. 